\title{
Bernd Michael
}

\section{Buridans moralphilosophische Schriften, ihre Leser und Benutzer im späten Mittelalter}

Wenn Johannes Buridan im Prooemium und in den einleitenden Quaestionen seines Kommentars zu den aristotelischen Ethica die Moralwissenschaft, die auch bei ihm gemäß dem seit den späten Peripatetikern gängigen Lehrstück in Ethik, Ökonomik und Politik dreigeteilt ist, als praktische Wissenschaft sowohl nach Gegenstand, Ziel und Methode von den anderen Wissenschaften unterscheidet, hat das ähnlich wie bei Aristoteles in der Zielsetzung des Werkes selbst gründende methodische Konsequenzen für den angesprochenen Hörerkreis der praktischen Philosophie. Die scientia moralis ist als Wissenschaft theoretischer Natur; ihrem Gegenstand nach aber, nämlich dem durch den Menschen Erwirkbaren, ist sie praktischer Natur, auf das Lenken menschlichen Handelns ausgerichtet und um des Tuns willen erworben: „Hoc viso“, so Buridan, „dico quod scientia moralis debet dici practica, quia est de operabilibus a

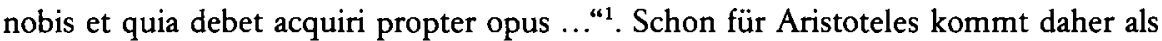
Hörer der Ethik nur derjenige in Frage, der über „Erfahrung im praktischen Leben“ verfügt und eine „gute Charakterbildung“ bereits mitbringt ${ }^{2}$. Auch nach Buridan ist die scientia moralis nur dem angemessen, der frei geboren (,ingenuus“) ist und durch seine Stellung hervorragt („excellens") ${ }^{3}$. Dem Jüngling dagegen, also dem Studenten und Schüler, so dürfen wir ergänzen, sind zum einen die Prinzipien der Moralwissenschaft "propter inexperientiam actuum humane vite "4 nicht evident, und zum anderen ist sein moralisches Handeln unter elterlichem und erzieherischem Zwang im eigent-

1 Johannes Buridanus, Quaestiones super decem libros Ethicorum Aristotelis, lib. I, qu. 2 (Paris 1513, Neudruck Frankfurt am Main 1968) $3^{\text {va }}$. Zusammenstellung der weiteren Drucke: Bernd Michael, Johannes Buridan: Studien zu seinem Leben, seinen Werken und zur Rezeption seiner Theorien im Europa des späten Mittelalters (Phil. Diss. FU Berlin 1978, Berlin 1985) 862-863 (im folgenden zitiert: Michael, Buridan). Der Druck Paris 1513 ist dort allerdings durch einen Fehler beim Setzen unterschlagen worden; dessen Neudruck sowie die Existenznachweise sind fälschlich der Inkunabel GW 5752 subsumiert worden. Zum Gegenstand der Ethik als praktischer Wissenschaft bei Buridan s. Gerbard Krieger, Der Begriff der praktischen Vernunft nach Johannes Buridanus (Münster 1986) 247-300.

${ }_{2}$ Georg Wieland, Ethica - scientia practica. Die Anfänge der philosophischen Ethik im 13. Jahrhundert (Münster 1981) 56-57. Vgl. Aristoteles, Nikomachische Ethik I 1, 1095 a 2 ff. und I 2, 1095 b 4 ff. Übers. Franz Dirlmeier (Berlin 1956) 7-8; Günther Bien, Die Grundlegung der politischen Philosophie bei Aristoteles (Freiburg-München 1973) 124-126.

${ }^{3}$ Buridan, Qq. Eth. (wie Anm. 1), lib. I, qu. 2, $3^{\text {vb }}$.

${ }^{4}$ Ebd. lib. I, qu 7, $7^{\text {va }}$ 
lichen Sinne nicht frei gewählt und unterliegt daher nicht allein seiner Selbstgesetzgebung, so daß er nicht zu den eigentlichen Adressaten der Ethik gehört. Wenn es unter dieser Voraussetzung für ihn dennoch sinnvoll und nützlich ist, sie zu hören, dann gründet dies - der theoretischen und praktischen Natur der Moralwissenschaft entsprechend - darin, daß ihre Prinzipien „ex doctorum auctoritate“ wie auch „ex audiendi consuetudine“ für den Jüngling Glaubwürdigkeit - also nicht Evidenz - haben und sein Handeln "sub debito regimine“ - also nicht frei - zur Tugend disponieren und vor dem Laster bewahren ${ }^{5}$. $\mathrm{Ob}$ das vom philosophus und von seinem Interpreten intendierte Publikum im Spätmittelalter tatsächlich erreicht wurde und in welchem Umfang die in der Moralwissenschaft selbst gründende Um- und Eingrenzung der Hörerschaft wirksam wurde, wird, wenn die texthistorischen Voraussetzungen und die institutionellen Rahmenbedingungen ihrer Rezeption seit dem 13. Jahrhundert zur Sprache gekommen sind, in Umrissen darzustellen sein.

Die Aristoteles-Rezeption, die den christlichen und lateinischen Westen nicht nur mit dem Zustrom griechisch-arabischer Textmassen, sondern auch mit einem umfassenden Konzept von Rationalitāt und einem kohärenten Bild der Welt konfrontierte, hatte ihre stürmischste Entwicklungsphase bereits hinter sich, als die für das spätere Mittelalter maßgeblichen Übersetzungen der praktischen Philosophie des Aristoteles entstanden. Robert Grosseteste hat seine Übertragung oder, wie Gauthier annimmt, seine Revision der älteren Ethik-Übersetzung 1246-1247 abgeschlossen ${ }^{6}$; Wilhelm von Moerbeke hat wohl unter Verwendung einer eigenen älteren Teilübersetzung die vollständige lateinische Version aller acht Bücher der „Politik“ um 1260 beendet $^{\gamma}$. Die ältere Übersetzung der pseudo-aristotelischen Ökonomik ins Lateinische entstand vor 1295, die jüngere recensio Durandi im Jahre $1295^{\circ}$. Die Úbersetzung der libri morales allein allerdings sagt über ihre tatsächliche Rezeption noch nicht viel aus. Erst die Kommentierung der neuen Texte gibt Aufschluß darüber. Die ältesten, bisher bekannt gewordenen Kommentare zu den Ethica scheinen dank der bis in das Ende des 12. Jahrhunderts zurückreichenden älteren Übersetzungen aus den dreißiger Jahren des 13. Jahrhunderts zu stammen'.

Der institutionelle Ort dieser Aneignung der philosophischen Ethik im lateinischen Westen ist - zunächst allein - die Pariser Universität, wo sie gemessen an den Kern-

${ }^{5}$ Ebd.

6 Renatus Antonius Gautbier, Praefatio, in: Aristoteles Latinus XXVI, 1-3. Fasc. 1: Ethica Nicomachea (Leiden-Bruxelles 1974) CLXXXVII, CCI; s. auch Wieland, Ethica 34-40.

${ }^{7}$ Jean Dunbabin, The Reception and Interpretation of Aristotle's "Politics“, in: The Cambridge History of Later Medieval Philosophy, ed. Norman Kretzmann, Antbony Kenny, Jan Pinborg (Cambridge 1982) 723.

${ }^{8}$ Hermann Goldbrunner, Durandus de Alvernia, Nicolaus von Oresme und Leonardo Bruni. Zu den Übersetzungen der pseudo-aristotelischen Ökonomik, in: Archiv für Kulturgeschichte 50 (1968) 200-239.

9 Wieland, Ethica 44-51. Zu den Kommentaren zu den „Politica“ und „Oeconomica“ vgl. Christoph Flüeler, Mittelalterliche Kommentare zur „Politik“ des Aristoteles und zur pseudo-aristotelischen „Ökonomik“, in: Bulletin de philosophie médiévale 29 (1987) 193-229. Unbefriedigend und vielfach irrig: Anna Stomczyńska, Repertorium commentariorum in Aristotelis Economica Latinorum quae in bibliothecis Europaeis asservantur, in: Mediaevalia Philosophica Polonorum 28 (1986) 167-200. 
stücken des Curriculums, nämlich der aristotelischen Logik und Naturphilosophie, von der Mitte des 13. Jahrhunderts bis in Buridans Zeit hinein nur von untergeordneter Bedeutung war. In Paris wurden die Ethica, später auch die Politica und Oeconomica bis in das 15. Jahrhundert hinein in knapp bemessener Vorlesungsdauer immer „extraordinarie“ gelesen und damit von den „ordentlichen“ Vorlesungen des „magistraliter" lesenden „magister regens" abgegrenzt. „Extraordinarie“ bedeutet, daß die zu hörenden Bücher in der Regel nicht zum Kanon der für den Erwerb der Grade vorgeschriebenen Textbücher gehörten; sie durften an den Tagen, für die kein allgemeines Vorlesungsverbot galt, nur außerhalb bestimmter Tageszeiten gelesen werden, und $z$ war nach den „ordentlichen“ Vorlesungen der Magister und nicht während der Predigten. $\mathrm{Zu}$ ihrer Abhaltung waren nach Genehmigung durch die Nation oder Fakultät Magister und Bakkalaurei berechtigt. Die Kleiderordnung war nicht so streng wie bei den ordentlichen Vorlesungen. Ein Ort war nicht vorgeschrieben; sie konnten also auch in den Häusern der Doktoren stattfinden. In der Mitte des 14. Jahrhunderts jedoch machte sich, auch wenn sich im Grundsätzlichen nicht allzuviel änderte, eine gewisse Höherbewertung der praktischen Philosophie bemerkbar: Die Pariser Statuten von 1366 schreiben immerhin Vorlesungen über den größeren Teil der Ethica für die Lizentiaten vor, die das Magisterium in artibus anstrebten. Die für die Rezeption der Schriften Buridans so wichtig werdenden, seit der Mitte des 14. Jahrhunderts neugegründeten Universitäten Mitteleuropas folgten in den Grundzügen weitgehend dem Pariser Vorbild. Die Ethica, oft auch die Oeconomica und die Politica, werden in ihren Studienordnungen vielfach zu den "ordinarie“ zu lesenden Büchern gerechnet; alle drei Schriften werden ferner insgesamt oder zum Teil an den bedeutendsten Universitäten ohne Ausnahme für den Erwerb der Grade, sei es der „licentia“, sei es des "magisterium in artibus" vorgeschrieben. Die Vorlesungsdauer betrug für die „Ethica“ in der Regel neun Monate, für die Oeconomica meist drei bis vier Wochen und für die Politica im allgemeinen vier bis sechs Monate. In diesen Statuten wird zwar die gegenüber Logik und Naturphilosophie geringere Wertschätzung der praktischen Philosophie nicht aufgehoben, gleichzeitig aber wird ihre erreichte Stellung fixiert und damit die Voraussetzung für eine intensivere Beschäftigung mit ihr und für die Rezeption von Buridans Werk im 14. und 15. Jahrhundert geschaffen ${ }^{10}$.

Die handschriftliche Überlieferung und die frühen Drucke der Werke Buridans schrieben dieser, wie es ein Amtsträger der Pariser Universität einmal pathetisch formulierte, „zweiten Sonne der Picardie “11 Kommentare zu allen drei Disziplinen der praktischen Philosophie zu. Bei genauerer Betrachtung jedoch zeigt sich, daß zwei dieser Kommentare mit Sicherheit nicht als authentisch bezeichnet werden können. Die Buridan in einem Berliner Codex zugeschriebene „Expositio in libros Oeconomicorum" ist nichts anderes als eine leicht gestraffte Fassung der in zahlreichen Handschriften überlieferten, wahrscheinlich 1359 entstandenen und irrtümlich unter dem Namen Alberts des Großen edierten „Expositio“ des Pariser Magisters und späteren

10 Nachweise bei Michael, Buridan 808 Anm. 5, 863-869.

11 Auctarium Chartularii Universitatis Parisiensis, ed. Henricus Denifle, Aemilius Chatelain u.a., Bd. 4 (Paris 1938) 314 (1481 Oktober 2). 
Halberstädter Bischofs Albert von Sachsen ${ }^{12}$. Etwas komplizierter sind die quellenkritischen Fragen, die die in einigen alten Drucken Buridan zugeschriebenen Quaestionen zu den „Politica“ aufwerfen. Der älteste Druck und die Handschriften, die das Werk überliefern, sind bis auf eine Ausnahme, über die zu sprechen sein wird, anonym. Allerdings scheint es in der zweiten Hälfte des 15. Jahrhunderts zwei Handschriften gegeben zu haben, die vermutlich diesen Kommentar Buridan zuweisen. Ein nachträglicher, aber offenbar alter Eintrag in einer kürzlich entdeckten Pariser Handschrift aus dem Ende des 14. Jahrhunderts schreibt dagegen diese Quaestionen, wie Flüeler gezeigt hat, unzweideutig dem um 1380 lehrenden Pariser Magister Nicolaus de Waldemonte zu. Da werkimmanente Gründe schon seit langem den Verdacht nahelegten, daß dieser Quaestionen-Kommentar in der vorliegenden Form nicht von Buridan stammen kann, da er nach dem Dezember 1360 entstanden sein muß, also wahrscheinlich nach Buridans Tod, wird eine Analyse der Moralphilosophie Buridans und seines Publikums auf diesen Kommentar gänzlich verzichten müssen ${ }^{13}$. Im Gegensatz dazu ist die Authentizität von Buridans Quaestionen-Kommentar zu den Ethica unbestritten. Dieses Werk, das unvollendet im zehnten Buch abbricht, da, wie es in einem Teil der Úberlieferung heißt, der Tod den Autor an der Vollendung seines Werkes gehindert habe, liegt, soweit man das vor dem Erscheinen einer kritischen Edition sagen kann, nur in einer Textrezension vor, die allerdings nicht in einem Zug, sondern über einen längeren Zeitraum hinweg gleichsam abschnittweise entstanden zu sein scheint. Die Anfänge dieser Vorlesung, deren etappenweises Voranschreiten sich im wesentlichen aus der oben beschriebenen Stellung der Ethik im Curriculum der Pariser Artistenfakultät erklären dürfte, reichen offenbar bis in die Zeit um 1340 zurück $^{14}$. Die Rezeptionsgeschichte der praktischen Philosophie Buridans im späten Mittelalter ist daher nichts anderes als die Geschichte der Leser und Benutzer nur eines einzigen seiner Werke, seiner Quaestionen zu den Ethica.

Die Bedeutung und das Gewicht dieses Buridanschen Werkes für die spätmittelalterliche Moralphilosophie können einige Zahlen über die erhaltenen Handschriften der Quaestionen verdeutlichen; denn ein häufig kopierter Text ist immer auch ein Text, der Erfolg und Einfluß hatte. Buridans Quaestionen zu den Ethica ${ }^{15}$ sind nach gegenwärtigem Kenntnisstand in 91 Codices bis heute erhalten geblieben; davon überliefern das Werk 54 Handschriften vollständig oder fast vollständig, 27 Handschriften zu großen Teilen, also meist Buch I-V oder Buch VI-X, und zehn Handschriften nur Fragmente und Exzerpte ${ }^{16}$. Buridans Ethik-Kommentar ist damit unge-

12 Michael, Buridan 928-933.

13 Michael, Buridan 888-896 ist im Lichte der Entdeckungen Cbristoph Flüelers zu ergänzen und zu korrigieren, vgl. in diesem Band 127-138.

14 Vgl. Michael, Buridan 870-876.

15 Auf die Behandlung der in vier bzw. fünf, teils anonymen, teils zugeschriebenen Manuskripten überlieferten „Expositio textus“ des Werkes kann hier verzichtet werden. Die Zusammenstellung der Handschriften bei Micbael, Buridan 824-828 ist zu erweitern um: Padova, Bibl. Antoniana, Ms. 415 Scaff. XIX, $1^{r}-92^{r}$ (= Buridan, Werke Nr. 27.12).

${ }^{16}$ Zusammenstellung der erhaltenen Handschriften nach dem Kenntnisstand von 1978 bei Michael, Buridan 829-862. Von der dort gegebenen Liste der Handschriften sind auf Grund seither angestellter Untersuchungen drei Codices zu streichen (Basel, UB, F.V. 5; München, BSB, Clm 
fähr in einem Drittel aller überhaupt erhaltenen Buridan-Handschriften überliefert. Einige Vergleiche können die Tragweite dieser Zahlen erhellen: Grossetestes vollständige Ethik-Übersetzung ist nach Gauthier in $279^{17}$, Alberts des Großen publizierter Ethik-Kommentar nach Fauser in $30^{18}$ und die „Sententia libri Ethicorum“ des Thomas von Aquin nach der Leonina in 126 jeweils vollständigen und fragmentarischen Handschriften überliefert ${ }^{19}$. Gemessen an den Zahlen der erhaltenen Kopien, ist Buridans Kommentar zwar nur in einem Drittel der Gosseteste-Handschriften, aber im Dreifachen der Albert-Handschriften erhalten. Bezeichnend aber ist der nur geringe Unterschied der Zahlen im Verhältnis zum Thomas-Kommentar (91:126). Buridan war also für sein spätmittelalterliches Publikum, was gern übersehen wird, nicht nur ein bedeutender Naturphilosoph, sondern vor allem einer der führenden Moralphilosophen der Zeit.

\section{Fortsetzung Fußnote von Seite 142}

11478; Wien, ÖNB, CVP 5316; zu den Verfassern der in diesen Handschriften überlieferten Ethik-Kommentare s. unten Anm. 35 und Anm. 40), zwölf Codices aber zu ergänzen: 1) "Basel, UB, F. I. 30, $2^{\mathrm{v}}$ (Fragment: Tituli quaestionum - Hs.: 14. Jh.; Paris (?), spăter Köln und Basel); 2) "Innsbruck, Stiftsbibliothek Wilten, Cod. XXXII. C. 9, $139^{\text {ra }}-305^{\text {ra }}$ (lib. I-V - Hs.: um 1400; Universität Wien); 3) "Leipzig, UB, Cod. 1448, 233 ${ }^{\mathrm{va}}-236^{\mathrm{rb}}, 238^{\mathrm{vab}}$ (Quaestionenverzeichnis und Fragment von lib. I, qu. 21 - Hs.: 15. Jh., 1. Hälfte; Universität Leipzig, mit Einband des Johannes Wetherhan); 4) (ehemals laut Katalog) Lübeck, Stadtbibliothek, Ms. philos. 3, 1'-262 (lib. I X,5, Prolog fehlt - Hs.: 15. Jh.); 5) "München, BSB, Clm 7479, 22 $2^{\text {ra }}-282^{\text {ra }}$ (lib. VI,1-X,5 - Hs.: 15. Jh. (1424); Deutschland, später Stift Indersdorf); 6) Notre Dame (Indiana, USA), Notre Dame Memorial Library, Ms. 22, 1'-258v (lib. 1,1-X,5 - Hs.: 15. Jh.; Italien; aus dem Besitz des Kardinals Ludovico Podocatoro, ca. 1430-1504); 7) Padova, BU, Cod. 1472, $1^{\mathrm{ra}}-158^{\mathrm{vb}}$ (lib. I,1-X,5 Hs.: 15. Jh. (1407); Universität Padua; spätere Besitzer: Pico della Mirandola (?), Kardinal Domenico Grimani, gest. 1523); 8) Sankt Paul im Lavanttal (Kärnten), Cod. Hosp. chart. 57 (vermutlich lib. I-X dieses Werkes - Hs.: 14. Jh. (1382); Vorbesitzer: Stift Spital am Pyhrn); 9) "Stuttgart, WLB, Cod. theol. et philos. fol. 161, $2^{\text {ra }}-467^{\text {va }}$ (lib. I,1-X,6 - Hs.: 15. Jh. (1466); im Jahre 1466 als Geschenk des Heilbronner Patriziers Alban Merklin an das unter seiner Pflegschaft stehende Karmeliterkloster „Unserer lieben Frau zu den Nesseln“ außerhalb der Stadtmauern); 10) "Città del Vaticano, BAV, Pal. lat. 1025, $1^{\mathrm{ra}}-168^{\mathrm{vb}}$ (lib. I,1-V,14 Anfang - Hs.: 15. Jh., 1. Hälfte; aus dem Besitz des Magisters Johannes Ambrosii aus Günzburg an die Heidelberger Artistenfakultät); 11) "Wertheim, Evangelische Kirchenbibliothek, Cod. 489, [1] $]^{\mathrm{ra}}-[249]^{\mathrm{va}}$ (lib. I,1-VI,22; VI,11-VI,15 fehlen - Hs.: 15. Jh., 1. Viertel; Süddeutschland, vermutlich aus dem Besitz des Kölner Professors der Theologie Konrad Wellin); 12) "Wien, ÖNB, CVP 5431, 6 $6^{\text {ra }}-291^{\text {vb }}$ (lib. I,1-X,6 - Hs.: 14. Jh., 2. Hälfte; Univ. Prag („reportate Prage"); späterer Besitzer: Artistenfakultät der Universität Wien). Die durch " gekennzeichneten Handschriften konnten eingesehen werden; auf Literaturangaben zu den einzelnen Codices und zu den Provenienzen muß hier allerdings verzichtet werden. Vgl. aber Mieczystaw Markowski, Die Rezeption Johannes Buridans Kommentars zur „Nikomachischen Ethik" des Aristoteles an den mitteleuropäischen Universitäten angesichts der in den Bibliotheken in Erfurt, Göttingen, Krakau, Kremsmünster, Leipzig, Melk, München, Salzburg, Wien, Wrocław und im Vatikan erhaltenen Handschriften, in: Mediaevalia Philosophica Polonorum 27 (1984) 89-131.

17 Gauthier, Praefatio (wie Anm. 6) CLII-CLXVIII.

${ }_{18}$ Winfried Fauser, Die Werke des Albertus Magnus in ihrer handschriftlichen Überlieferung. Teil 1: Die echten Werke (Münster 1982) 175-178; Winfried Fauser, Albertus-Magnus-Handschriften. 3. Fortsetzung, in: Bulletin de philosophie médiévale 26 (1984) 142-143.

19 Thomas de Aquino, Opera omnia 47,1 (Rom 1969) $1^{*}-30^{*}, 36^{*}$. 
Der Rezeptionsprozeß des Werkes durch das spätmittelalterliche Publikum verläuft nicht kontinuierlich und ist geographisch nicht einheitlich. Von den 91 erhaltenen Handschriften des Ethik-Kommentars gehören 42 in das 14. und 49 in das 15. Jahrhundert. Eine genauere Aufschlüsselung dieser Zahlen zeigt, daß drei Handschriften vor 1375,31 von $1376-1400,15$ von $1401-1425$ und je sieben von $1426-1450$ und 1451-1500 entstanden. Acht Handschriften sind allgemein der zweiten Hälfte des 14. Jahrhunderts und 16 Codices der ersten Hälfte des 15. Jahrhunderts zuzurechnen. Der Befund ist eindeutig: Die Zahl der Kopien steigt im letzten Viertel des 14. Jahrhunderts sprunghaft an und verhart trotz eines Tendenzumschwungs um $1400 \mathrm{im}$ ersten Viertel des 15. Jahrhunderts weiterhin auf einem hohen Niveau, um dann seit dem zweiten Viertel des 15 . Jahrhunderts deutlich abzunehmen.

Leser und Publikum fanden Buridans Quaestionen zu den Ethica bis in den Anfang des 16. Jahrhunderts in den meisten Ländern des katholischen Europa. Dennoch war der Einfluß nicht überall gleich intensiv. Die Verteilung der Kommentar-Handschriften nach den heutigen Bibliotheksorten ist zwar ein grober, aber dennoch aufschlußreicher Indikator: In Frankreich und Belgien befinden sich 16, in Mitteleuropa (in Deutschland, Österreich und Polen, in der Schweiz und der Tschechoslowakei) 54, in Italien 17 und in Schweden zwei Handschriften, in England und den USA je ein Codex, in Spanien und Portugal keiner.

Der institutionelle Ort von Rezeption und Kommentierung der praktischen Philosophie im lateinischen Westen war von Anfang an, wie gezeigt wurde, die Universität. Die literarische Form der Kommentierung - lectio und quaestio - entsprachen in $\mathrm{Me}$ thode und Ziel der Institution ${ }^{20}$. Auch Buridans Quaestionen-Kommentar zu den Ethica ist daher schon von der Form her universitäre Fachliteratur par excellence. Sein Publikum, seine Leser wie seine Benutzer, muß das Werk, so will es scheinen, an den Universitäten finden. Es ist natürlich unmöglich und unnötig, die Details des Rezeptionsprozesses seines Ethik-Kommentars an den spätmittelalterlichen Universitäten hier auszubreiten. Nur einige Wegmarken und Aspekte möchte ich hervorheben.

An der Pariser Universität scheint der über den unmittelbaren Scholaren-Kreis Buridans hinausgehende Einfluß seines Kommentars erst nach 1360, also nach seinem Tod begonnen zu haben. Das unvollendete Werk scheint, modern ausgedrückt, erst aus dem Nachlaß herausgegeben worden zu sein. Das älteste datierte, vielleicht das älteste erhaltene Manuskript überhaupt ist in den Jahren 1362-1363 vermutlich in Paris vollendet worden ${ }^{21}$. Spätestens seit den siebziger Jahren des 14. Jahrhunderts begannen die Magister der Pariser Fakultäten, vor allem die Artisten und Theologen, das Werk verstärkt abzuschreiben, vor allem aber sich abschreiben zu lassen oder fertige Kopien zu erwerben. Kollegien wie das Collège Dormans-Beauvais, das Collège de

20 Bernardo C. Bazàn, Les questions disputées, principalement dans les facultés de Théologie, in: Les questions disputées et les questions quodlibétiques dans les facultés de Théologie, de Droit et de Médecine (Typologie des sources du moyen âge occidental 44-45, Turnhout 1985) 25-40. $21{ }^{*}$ Città del Vaticano, BAV, Urb. lat. 198, $2^{\text {ra }}-199^{\text {ra }}$. Diese Handschrift ist von mehreren, teils wohl englischen, teils wohl aber auch französischen Händen 1362-1363 geschrieben worden, wie einige Datierungen am Rand und am Schluß $\left(164^{r}, 166^{r}, 199^{\top}\right)$ zeigen. 
Navarre und die Sorbonne schlossen sich $a^{22}$. Bemerkenswert unter den nach Schrift und Ausstattung in Frankreich, wohl in Paris entstandenen Handschriften ist die große Zahl an Pergamenthandschriften, die mit ihrem Fleuronnée- und Initialschmuck das übliche Maß der universitären Gebrauchshandschriften weit übertreffen und den sozialen Status ihrer Auftraggeber und Besitzer reflektieren, unter denen sich gelehrte Kanoniker der Kathedralen von Chartres, Reims und Cambrai ebenso befanden wie der Abt der Benediktinerabtei Marchiennes bei Douai ${ }^{23}$.

Vor diesem Hintergrund wird die besondere Wertschätzung verständlich, der sich Buridans Kommentar in dem um Johannes Gerson sich gruppierenden Zirkel erfreut zu haben scheint: Henri Chicot, Schüler des Collège de Navarre wie Gerson und ihm eng verbunden, hat sich das Werk 1389 abschreiben lassen ${ }^{24}$. Gerson selbst, für den Buridan ein Mann war, „qui multa vidit, legit atque conscripsit", sieht in ihm und seinem Ethik-Kommentar im Jahre 1400 einen Weggefährten in seinem eigenen Kampf gegen die „curiositas" der Professoren ${ }^{25}$. Es dürfte daher sicherlich einmal nützlich sein, die Beziehungen zwischen der Naturrechtstheorie Buridans und der Gersons insbesondere im Hinblick auf die für die Kirchenreform so wichtige Frage der Epikie näher zu untersuchen ${ }^{26}$. Dem Erfolg des Buridanschen Oeuvres beim Pariser Universitātspublikum um 1400 folgte spätestens um 1410 mit dem, was die Pariser Nominalisten 1474 die „tertia persecutio nominalium“ nannten, der jähe Sturz in die Tiefen des Verschweigens und Vergessens ${ }^{27}$, der sein endgültiges Ende erst mit der Wiederentdeckung Buridans im letzten Viertel des 15. Jahrhunderts fand. Schon zuvor war allerdings die in Paris untergegangene "Sonne der Pikardie“ anderswo längst wieder aufgegangen.

Das Reich war nicht nur ein Reich ohne Hauptstadt, es war bis in die Mitte des 14. Jahrhunderts auch ein Reich ohne Universitäten und damit ohne ausgeprägte eigene wissenschaftlich-philosophische Schultraditionen. Universitätsgründungen wie Prag (1348) ahmten daher nicht nur institutionell, sondern auch intellektuell ihr Vorbild nach. Die Prager Universität ${ }^{28}$, die nach einer Konsolidierungsphase offenbar erst

22 Michael, Buridan 326-327. Aus der Bibliothek der Sorbonne stammen *Paris, BN lat. 16128 und lat. 16129.

${ }^{23}$ Vgl. z. B. die Handschriften "Auxerre, BM 232; "Cambrai, BM 165; "Chartres, BM 283 (Hs. im 2. Weltkrieg schwer beschädigt); 'Douai, BM 692 (mit dem Wappen des 1412 gestorbenen Guillaume Chrétien, der als Mönch in Tournai, als Bakkalaureus der Theologie in Paris und als Abt in Marchiennes nachweisbar ist); 'Paris, Bibl. Mazarine 3515; "Reims, BM 889.

${ }^{24}$ Handschrift: "Chartres, BM 283; Michael, Buridan 325 Anm. 117.

${ }^{25}$ Jean Gerson, Oeuvres complètes, ed. P. Glorieux, Bd. 2 (Paris 1960) 32.

${ }^{26} \mathrm{Zu}$ Buridans Positionen vgl. Buridan, Qq. Eth. (wie Anm. 1), lib. V, qu. 26-27, 112 $2^{\mathrm{vb}}-114^{\mathrm{rb}}$; Krieger, Begriff der praktischen Vernunft 139-145. Zu Gersons Auffassungen vgl. Louis B. Pascoe, Jean Gerson. Principles of Church Reform (Leiden 1973) 65-68.

27 Franz Ebrle, Der Sentenzenkommentar Peters von Candia, des Pisaner Papstes Alexanders V. (Franziskanische Studien, Beiheft 9, Münster 1925) 324. Zur Chronologie und zu den Hintergründen der Auseinandersetzungen am Beginn des 15. Jahrhunderts vgl. Zénon Kaluza, Les querelles doctrinales à Paris. Nominalistes et réalistes aux confins du XIV et du XVe siècles (Quodlibet 2, Bergamo 1988).

${ }^{28}$ Peter Moraw, Die Universitāt Prag im Mittelalter. Grundzüge ihrer Geschichte im europäi- 
um 1360 organisatorisch festere Formen fand, wurde so zur Drehscheibe des Pariser Buridanismus in Mitteleuropa. Die Fernwirkungen reichten bis Schweden ${ }^{29}$. Seit dem Anfang der siebziger Jahre des 14. Jahrhunderts waren Buridans Ethica in Prag bereits bekannt; die ersten Kopien entstanden ${ }^{30}$. In diesem geistigen Klima wird es verständlich, weshalb Johann von Neumarkt, der Kanzler Karls IV., zur gleichen Zeit nachdrücklich sein Interesse an diesem Werk bekundete und wohl einen Magister der Theologie in einem aufschlußreichen Brief bat, ihm einen fähigen, mit den Abbreviaturen in den philosophischen Handschriften der Zeit vertrauten Schreiber - „aliquem ... intelligentem clericum qui legibilem textualem notulam sciat scribere quique breuiaturas loyce et philosophie bene et pertinenter cognoscat" - zu schicken, um von einem entliehenen Exemplar eine Kopie des Werkes des „magister famosus ... Buridanus" anzufertigen ${ }^{31}$. Bis in den Anfang des 15. Jahrhunderts, als in den an den Namen Hus' sich knüpfenden Auseinandersetzungen der Buridanismus in Prag ein ebenso abruptes Ende wie in Paris fand, entstand etwa ein Sechstel der heute noch erhaltenen Ethik-Handschriften Buridans in Prag oder nach Prager Vorlage. Buridan war tatsächlich, wie Heinrich Egher von Kalkar einmal bemerkte, „fons scientiarum Pragae ${ }^{\text {“32 }}$. Der ausgeprägt schulische Charakter der Buridan-Rezeption in Prag, der für die Rezeptionsgeschichte an den mitteleuropäischen Universitäten bestimmend bleiben sollte, zeigt sich in zwei Erscheinungen besonders deutlich, nämlich im Kopieren der Werke in Form des Mitschreibens nach Diktat sowie in der Kommentierung des Textbuches, also der aristotelischen Ethica, nach dem Buridanschen Modell. Die Prager Universität, die das Pecia-System nicht kannte, veranstaltete besondere „Vorlesungen“, im Detail durch Statuten geregelt, die „pronuntiatio“ und „reportatio“, in denen die maßgeblichen universitären Texte mündlich, nach Diktat tradiert wurden, so daß mehrere Scholaren nach ein und derselben "pronuntiatio“ schreiben und aus einer Diktierveranstaltung mehrere Reportationen gleichzeitig hervorgehen konnten. Von Buridans Ethik-Quaestionen sind drei am selben Tag beendete Prager Reportationen aus dem Jahre 1382 erhalten, von denen eine wiederum später als Vorlage für eine weitere Kopie gedient hat ${ }^{33}$. Die zweite Erscheinung, die in Prag in ihrer Anfangsphase zu beobachten ist, ist Ausdruck des Verlustes konzeptioneller philosophischer

\section{Fortsetzung Fußnote von Seite 145}

schen Zusammenhang, in: Die Universität Prag (Schriften der Sudetendeutschen Akademie der Wissenschaften und Künste 7, München 1986) 9-134.

29 Handschrift: Uppsala, UB, Cod. C 609.

30 Handschriften: Praha, SK, Cod. V. A. 25 (ca. 1368-1382); Uppsala, UB, Cod. C 609 (1374, 1379); Wroctaw, BU, Cod. IV. F. 2 (vor 1379); Kraków, BJ, Cod. 744 (um 1370/80); “Wien, ÖNB, CVP 5431 (ca. 1380).

31 Johann von Neumarkt, Briefe. Ges., hrsg. und erl. von Paul Piur (Vom Mittelalter zur Reformation 8, Berlin 1937) 207-208.

${ }^{32}$ Léopold Delisle, Un trait de la jeunesse de Buridan, in: Bulletin de la Société de l'histoire de Paris et de l'lle-de-France 2 (1875) 102.

${ }^{33}$ Handschriften: "Basel, UB, F. V. 3 ; "Leipzig, UB, Cod. 1447; 'Melk, Stiftsbibliothek, Cod. 542 (48; B 12). Spātere Kopie nach dieser Vorlage: "Basel, UB F.V. 4. Zur Sache vgl. Karl Christ, Petia. Ein Kapitel mittelalterlicher Buchgeschichte, in: Zentralblatt für Bibliothekswesen 55 (1938) 1-44; Gerbardt Powitz, „Modus scolipetarum et reportistarum“. Pronuntiatio and Fifteenth-Century University Hands, in: Scrittura e civilità 12 (1988) 201-211. 
Kraft und der Versteinerung des Lehrbetriebs. Die Textbücher, insbesondere auch die Ethica, werden nicht mehr selbständig, sondern in der Regel in der QuaestionenFolge und vielfach auch im Geiste nach dem Vorbild des Modell-Kommentars, nämlich dem Buridans, disputiert, so daß bis in unsere Tage Kommentierungen dieser Art von der Forschung gelegentlich für das Original gehalten worden $\operatorname{sind}^{34}$.

An der Wiener Universität, an der Buridans Ethik erstmals zwar auch schon in den siebziger Jahren des 14. Jahrhunderts gelesen wurde, setzt eine Intensivierung der Rezeption erst mit der Reorganisation der Universität nach 1384 ein. Im Gegensatz zu Paris und Prag bricht sie am Beginn des 15. Jahrhunderts nicht ab, sondern bleibt das ganze Jahrhundert über in breitem Strom wirksam. Die Wiener Artisten und Theologen schrieben das Werk jedoch nicht nur ab, sondern nach Prager Vorbild diente es ihnen als formales und inhaltliches Modell für ihre eigenen Vorlesungen zu den Ethica. Zu nennen sind hier unter anderem die nie näher untersuchten Wiener EthikKommentare von Urban von Melk, Jodocus Weiler von Heilbronn, Andreas von Schärding, Jakob und Thomas von Wuldersdorf, Wolfgang Kydrer und Andreas Wall aus Walsheim ${ }^{35}$.

Das für Prag und Wien geltende Muster der Buridan-Rezeption läßt sich, wenn auch teilweise weniger ausgeprägt, an Universitäten wie Erfurt und Krakau, Heidelberg, Leipzig und Rostock beobachten. Im Unterschied zu den beiden älteren Universitäten wird das Werk an den jüngeren Schwestern nicht mehr so häufig kopiert, was der Verfügung über den Text aber keinen Abbruch tut, da die älteren Kopien mit den Magistern wanderten und schließlich auf den Markt drängten, so daß man auch auf andere Weise als durch Abschreiben in den Besitz der Bücher gelangen konnte. In den eigenen Ethik-Vorlesungen dagegen folgt man weiterhin dem Buridanschen Modell, so Heinrich Toke $^{36}$ und ein anonymer Kommentator vermutlich in Erfurt ${ }^{37}$, so der

34 Micbael, Buridan 267-268.

35 Nachweise ebd. 346-347. Andreas von Schärdings Ethik-Kommentar ist außer in der dort genannten Handschrift auch in 'München, BSB, Clm $11478,1^{\mathrm{r}}-238^{\mathrm{v}}$ überliefert. Die sich stark an Buridans Quaestionen anlehnende Ethik-Vorlesung des aus Salzburg stammenden Wolfgang Kydrer dürfte in dem weitgehend eigenhändig geschriebenen Codex *München, BSB, Clm $19678,5^{r}-101^{r}$ erhalten sein. Über Kydrer vgl. Virgil Redlich, Tegernsee und die deutsche Geistesgeschichte im 15. Jahrhundert (München 1931) 41-45. Der Kommentar des Andreas Wall ist enthalten in "München, BSB, Clm 18883, $1^{\mathrm{r}}-225$; zu dem von Buridan abhängigen Ethik-Kommentar des Thomas Wölfel von Wuldersdorf, s. Charles H. Lobr, Medieval Latin Aristotle Commentaries. Authors: Robertus-Wilgelmus, in: Traditio 29 (1973) 189. - Zu den Anfängen der Rezeption in Wien vgl. die Handschrift Kraków, BJ 658: sie ist als Prager „reportatio“ nach einer Wiener Vorlage aus dem Jahre 1372 ("reportate Wienne") entstanden.

${ }^{36}$ Berlin, SBPK, Ms. lat. qu. 356, $1^{\mathrm{ra}}-117^{\mathrm{vb}}$. Zum Verfasser s. Erich Kleineidam, Universitas studii Erffordensis 1 (Leipzig ${ }^{2}$ 1985) 289-292 Nr. 31. Vgl. auch Michael, Buridan 353 Anm. 236.

37 Berlin, DSB, Ms. lat. qu. 991 (an: $4^{\circ}$ Inc. 3408, 15), $1^{\text {ra }}-124^{\text {rb }}$. Dieser anonyme und unvollständige Kommentar (lib. I,3-IV,2), der auf Grund der benutzten Papiere im 15. Jahrhundert, 2./ 3. Viertel, entstanden ist und sich in einem Band befindet, der 1907 wahrscheinlich aus der Gymnasialbibliothek Heiligenstadt (im Eichsfeld) an die Berliner Bibliothek gelangte, weist durch seinen Einband aus der Werkstatt des Nicolaus von Havelberg auf Erfurter Provenienz. Zu dieser Erfurter Werkstatt s. Ernst Kyriss, Deutsche Buchbinder der Spätgotik und Renaissance, in: Archiv für Geschichte des Buchwesens 3 (1961) 186 Nr. 34. 
Leipziger und ehemalige Prager Magister Petrus von Prenzlau ${ }^{38}$, so Nicolaus von Amsterdam, die aus Erfurt gekommene überragende Gestalt der Rostocker Artistenfakultä $^{39}$. Der interessanteste Autor unter all diesen Kommentatoren ist - von den Historikern bisher übersehen - der Heidelberger Artist, Konzilstheologe, königliche Rat und spätere Regensburger Bischof Konrad Koler von Soest, dessen Buridan folgender Kommentar zu den Ethica, soweit ich sehe, in sechs Handschriften erhalten ist ${ }^{40}$.

Buridans Ethik-Kommentar fand sein Publikum jedoch nicht nur in Frankreich und in den Ländern der Mitte Europas, sondern zu großen Teilen auch in Italien. Im Unterschied aber zum Reich war Italien ein Land, das auf seit langem etablierte Universitäten und auf gut begründete wissenschaftliche Traditionen zurückblicken konnte, so daß der Buridanismus dort nie ein solches Gewicht erlangen konnte, das dem im wissenschaftlich weitgehend traditionslosen Mitteleuropa vergleichbar war.

${ }^{38}$ Die Buridan folgenden „Disputata Ethicorum“ des bis 1433 nachweisbaren Prager und späteren Leipziger Magisters Petrus (Weggun) de Prenslavia (aus Prenzlau, Uckermark) sind erhalten in "Wolfenbüttel, HAB, Cod. Guelf. 805 Nov., $161^{\mathrm{r}}-288^{\mathrm{vb}}$. Zum Autor und zu einem Teil seiner Werke s. Josef Tríska, Repertorium biographicum Universitatis Pragensis praehussiticae 13481409 (Praha 1981) 466.

39 Seine dem Modell Buridans bis in die Besonderheit des Textabbruchs in lib. X, qu. 5 folgenden Quaestionen zu den Ethica sind außer in den bei Charles H. Lobr, Medieval Latin Aristotle Commentaries. Authors: Narcissus-Richardus, in: Traditio 28 (1972) 305 genannten Handschriften in mindestens drei weiteren Codices anonym überliefert: 1) "Leipzig, UB, Cod. 1448, $2^{\text {ra }}$ $231^{\text {va }}$ (lib. I-X; schon im 15. Jh. auf dem Einband und 68 $8^{\mathrm{r}}$ Buridan zugeschrieben); 2) Wien, Dominikanerkloster, Cod. 79/44, $2^{\text {ra }}-196^{\text {vb }}$ (lib. I-VII); 3) "Zeitz, Domherrenbibliothek, Cod. LVIII (21), $1^{\mathrm{ra}}-267^{\mathrm{ra}}$ (lib. I-X). Alle diese Handschriften entsprechen der Redaktion der bei Lohr genannten zugeschriebenen bzw. anonymen Nürnberger Handschriften und weichen daher deutlich von Lohrs Textspecimen nach der Handschrift "Leipzig, UB, Cod. 1451, $1^{\mathrm{ra}}-190^{\mathrm{vb}}$ ab, die ebenfalls Nicolaus von Amsterdam zugeschrieben ist. Die erheblichen Textunterschiede beider Handschriftengruppen bedürfen einer genaueren Untersuchung und Erklärung. Zum Autor s. Kleineidam, Universitas 1 (wie Anm. 36) 406-407.

${ }^{40}$ Über ihn vgl. Remigius Bäumer, Konrad von Soest, in: Neue deutsche Biographie 12 (Berlin 1980) 523-524; Winfried Eberbard, Konrad Koler von Soest, Konzilstheologe und königlicher Rat, in: Von Soest - aus Westfalen. Wege und Wirkung abgewanderter Westfalen im späten Mittelalter und in der frühen Neuzeit, hrsg. Heinz-Dieter Heimann (Paderborn 1986) 93-123. Auf seinen bis heute vielfach mit Buridans Werk verwechselten Ethik-Kommentar hat kurz hingewiesen Charles H. Lobr, Medieval Latin Aristotle Commentaries. Authors A-F, in: Traditio 23 (1967) 396. Die eindeutigen Zuschreibungen des Werkes in der Berliner und in der Münchener Handschrift erlauben nunmehr die Zusammenstellung der folgenden sechs Handschriften seiner Quaestionen zu den Ethica: 1) "Basel, UB, F. V. 5, $1^{\text {ra }}-413^{\text {vb }}$ (lib. I-V; mit alter Zuschreibung an Buridan); 2) "Berlin, SBPK, Ms. lat. fol. 783, $2^{\text {ra }}-334^{\text {va }}$ (lib. I-V); 3) "Gießen, UB, Hs. 623, $2^{\text {ra }}-$ $385^{\text {rb }}$ (lib. I-V; die versteckte Zuschreibung an Konrad von Soest in Gabriel Biels Titelbeschriftung „Questiones Ratisponenses super 5 primos libros Ethycorum“ vom Katalogbearbeiter nicht erkannt, s. Wolfgang Georg Bayerer, Handschriftenkataloge der Universitätsbibliothek Gießen 4 (Wiesbaden 1980) 24-25); 4) *München, UB, $2^{\circ}$ Cod. ms. 565, $1^{\mathrm{r}}-504^{\mathrm{r}}$ (lib. I,1-X,6, s. Natalia Daniel, Gerbard Schott, Peter Zabn, Die lateinischen mittelalterlichen Handschriften der Universitätsbibliothek München 3,2 (Wiesbaden 1979) 87-88; Miecislaus Markowski, Buridanica quae in codicibus manu scriptis bibliothecarum Monacensium asservantur (Wroclaw u. a. 1981) 110112); 5) "Wien, ÖNB, CVP 5316, $4^{\mathrm{ra}}-412^{\mathrm{rb}}$ (lib. I-X, s. auch Markowski, Rezeption (wie Anm. 16) 130-131, mit irriger Zuschreibung an Buridan); 6) Wien, ÖNB, CVP 5340, $1^{\text {ra }}-359^{\text {va }}$ (lib. V-VII, s. Miecislaus Markowski, Repertorium commentariorum medii aevi in Aristotelem Latinorum quae in bibliothecis Wiennae asservantur (Wrocław u.a. 1985) 118, 263). 
Wie auch anderswo waren es die Artistenfakultäten vor allem in Bologna und Padua, die die Buridan-Rezeption in Italien trugen. Die enge Verbindung von Philosophie und Medizin aber, die für diese Fakultäten charakteristisch war und die im nordalpinen Europa kein Äquivalent hatte, erklärt auch die Verzögerung, mit der Buridans Quaestionen zu den Ethica gegen Ende der achtziger Jahre des 14. Jahrhunderts, und damit knapp 25 Jahre nach Beginn der Rezeption der naturphilosophischen Werke der Pariser Schule, in Italien bekannt wurden ${ }^{41}$.

Vom Florentiner Kanzler und Humanisten Coluccio Salutati, der sich um 1400 darüber beklagt, daß es ihm trotz aller Mühen nicht gelungen sei, sein unvollständiges Exemplar der „quaestiones optimi Buridani“ zu den Ethica zu vervollständigen ${ }^{42}$, bis zu Pietro Pomponazzi, der kein Freund der ,secta nominalium“ war und sich 1520 sehr kritisch mit Buridans Kommentar auseinandersetzte ${ }^{43}$, reicht das Spektrum der Leser und Benutzer Buridans in Italien. Die ausschließliche Ausrichtung auf das universitäre Milieu wie in Deutschland und Böhmen fehlte. Während die naturwissenschaftlich, medizinisch und astrologisch orientierten italienischen Artisten und Mediziner Buridans Ethik-Kommentar im Vergleich zu seinen übrigen Werken nur ein relativ geringes Interesse entgegenbrachten, genoß derselbe Kommentar in humanistischen Kreisen Italiens seit 1400 hohes Ansehen, wie nicht nur das Beispiel Salutatis, sondern auch das des Florentiners Donato Acciajuoli zeigt, der wohl um die Mitte des 15. Jahrhunderts Buridans Kommentar mit großer Sorgfalt studiert hat ${ }^{44}$. Andere, die neuen geistigen Tendenzen ihrer Zeit vertretende Männer teilten dieses Interesse. Domenico de Capranica, Bischof von Fermo, Kardinal und Mentor von Enea Silvio Piccolomini, Bessarion, Pico della Mirandola sowie einige namentlich nicht bekannte, aber wohl humanistischen Kreisen an der Kurie zuzurechnende Männer, Päpste und bibliophile Kardinäle wie Ludovico Podocatoro († 1504), Domenico Grimani († 1523) und Niccolo Ridolfi († 1550) glaubten, auf Buridans Ethik-Kommentar in ihren Bibliotheken nicht verzichten zu können ${ }^{45}$. Auch die adligen Bibliophilen der italieni-

4 Vgl. die vermutlich oder mit Sicherheit in Italien entstandenen Handschriften *Città del Vaticano, BAV, Vat. lat. 2166 (a.d. 1388); Venezia, Biblioteca Marciana, Cod. 1984 (Zan. lat. 262) (a.d. 1394/96); Bologna, BU, Cod. lat. 366 (a.d. 1395); Berlin, SBPK, Ms. lat. fol. 934 (14. Jh., Ende).

42 Coluccio Salutati, Epistolario, ed. Francesco Novati, Bd. 3 (Roma 1896) 391-392 (lib. XI, epist. 16 - 1400 Mai 7).

${ }^{43}$ Petrus Pomponatius, Libri quinque de fato, de libero arbitrio et de praedestinatione, ed. $R$ ichard Lemay (Lucca 1957) 54-55, 239.

44 Firenze, Biblioteca Nazionale Centrale, Cod. II. I. 81 (=Strozz. in f. 189-90; Magl. Cl. XXI, 124-125). Vgl. Eugenio Garin, La circolazione in Italia, di pensatori quali Buridano ..., in: Giornale critico della filosofia italiana 37 (1958) 153.

4s Vgl. oben Anm. 16 Nr. 6 und 7 sowie die Handschriften *Città del Vaticano, BAV, Chigi A VI 189; Ross. lat. 785; Vat. lat. 2165; Vat. lat. 4557; Venezia, Biblioteca Marciana, Cod. 1984 (Zan. lat. 262). Das 1403-1410 in Ferrara entstandene Manuskript "Paris, BN lat. 6460 scheint nach Ausweis der alten Signaturen aus dem Besitz des Kardinals Niccolo Ridolfi (s. Bernard de Montfaucon, Bibliotheca bibliothecarum manuscriptorum nova 2 (Paris 1739) 780 Nr. 32) über die Bibliothek Katharinas von Medici in die Königliche Bibliothek in Paris gelangt zu sein. Zu diesen Zusammenhängen vgl. Henri Omont, Un premier catalogue des manuscrits grecs du Cardinal Ridolfi, in: Bibliothèque de l'Ecole des Chartes 49 (1888) 309-310. Zu Bessarions Handschrift vgl. 
schen Renaissance, die wie alle Bibliophilen eher das Erprobte als das Neue erwarben, zeigten Interesse an diesem Werk. Den Anfang machten schon vor 1426 die Visconti und Sforza in Mailand ${ }^{46}$; Federico da Montefeltro, der Herzog von Urbino, folgte in der zweiten Hälfte des 15. Jahrhunderts ${ }^{47}$. Das italienische Publikum von Buridans Moralphilosophie bestätigt, daß gerade die praktische Philosophie, die zum Fächerkanon der „studia humanitatis“ gehörte, es war, die zwischen humanistischer und scholastischer Kultur aller Polemik und Skepsis zum Trotz Koexistenz und Brückenschlag ermöglichte.

Der Einfluß von Buridans Moralphilosophie im Spätmittelalter war, so glaube ich gezeigt zu haben, beträchtlich. Die große Mehrzahl der Leser und Benutzer nicht nur seiner logischen und naturphilosophischen, sondern auch seiner moralphilosophischen Werke im 14., 15. und 16. Jahrhundert rekrutierte sich aus dem Universitätsmilieu. Ob in Frankreich oder Italien, ob in Böhmen, Deutschland oder Polen - zumeist waren es Scholaren, Magister und Doktoren der Universitäten, die seine Werke kopierten und benutzten, kauften und sammelten. Das Publikum, das sich seiner praktischen Philosophie zuwandte, bestand zum großen Teil aus Spezialisten und „Fachgelehrten“, aus Magistern und Doktoren, die das Werk für ihre eigene Lehre benutzten. Auch Buridans Ethik-Kommentar erweist sich daher nicht nur von Inhalt, Form und Entstehungsbedingungen, sondern auch von den Rezeptionsbedingungen her als gelehrte Fachliteratur, als Schulphilosophie, die sich an ein spezialisiertes Gelehrtenpublikum wandte; der Ethik-Kommentar unterscheidet sich in dieser Hinsicht nicht von seinen anderen Aristoteles-Kommentaren. Auf der anderen Seite aber gelang es diesem Werk, in einem gewissen Umfang Leser außerhalb des engen Zirkels des gelehrten Fachpublikums zu finden, wodurch es sich vom übrigen Oeuvre des Pariser Magisters abhebt. Es fand Leser, zumindest aber Besitzer in einem hochgebildeten Laienpublikum und im hohen Klerus. Bedeutende Parteigänger des Humanismus in Italien, mit Jakob Wimpheling aber auch in Deutschland ${ }^{48}$ besaßen dieses Werk eben-

\section{Fortsetzung Fußnote von Seite 149}

auch Lotte Labowsky, Bessarion's Library and the Biblioteca Marciana (Roma 1979) 229 Nr. 689, 281 Nr. 752, 312 Nr. 620(?), 355 Nr. 536, 395, 449, 469; zu Pico della Mirandolas Codex vgl. Calori Cesis, Giovanni Pico della Mirandola detto la Fenice degli Ingegni, cenni biografici (Memorie storiche della città e dell'antico ducato della Mirandola 11, Mirandola 1897) 58 Nr. 852; Pearl Kibre, The Library of Pico della Mirandola (New York 1936) 219 Nr. 746.

46 Elisabeth Pellegrin, La bibliothèque des Visconti et des Sforza, ducs de Milan, au XVe siècle (Paris 1955) 118 Nr. 201.

47 Aus seinem Besitz: "Città del Vaticano, BAV, Urb. lat. 198 und Urb. lat. 1367. Beide Handschriften sind nach dem Schriftbefund in Frankreich entstanden, zur älteren der beiden s. oben Anm. 21. Die jüngere Handschrift (Urb. lat. 1367) wurde unter Verwendung einiger älterer Faszikel (14. Jh., Ende) in der ersten Hälfte des 15. Jahrhunderts vervollständigt. Erst Federico da Montefeltro ließ sie dann am Anfang mit Initialen im Stil seiner Zeit und mit seinem Wappen schmücken.

${ }_{48} \mathrm{Zu}$ Wimphelings Verteidigung Buridans gegenüber Jakob Locher Philomusus vgl. Michael, Buridan 314. $\mathrm{Zu}$ weiteren Details und zum Hintergrund dieser Auseinandersetzungen s. James H. Overfield, Humanism and Scholasticism in Late Medieval Germany (Princeton 1984) 185-207, bes. 202; Johannes Helmrath, „Humanismus und Scholastik“ und die deutschen Universitäten um 1500, in: Zeitschrift für historische Forschung 15 (1988) 187-203. Auch der von Locher an- 
so wie einige der angesehensten adligen Büchersammler Italiens. Konfrontiert man das tatsächliche, an Hand der erhaltenen Handschriften erkennbare Publikum des Kommentars mit dem bei Aristoteles und Buridan selbst anvisierten Hörerkreis, wie ich ihn zu Beginn dieses Vortrages definiert habe, dann zeigt sich deutlich, daß die beabsichtigte Eingrenzung des Publikums der Moralwissenschaft auf die im praktischen Leben Erfahrenen, die sie um des Tuns willen erwerben, nur von mäßigem Erfolg gekrönt war. Nicht die praktische, sondern die theoretische Natur der scientia moralis Buridans macht ihren Erfolg beim spätmittelalterlichen Publikum aus. Schule“, nämlich Konrad von Soests Kommentar (München, UB, $2^{\circ}$ Cod. ms. 565, s. oben Anm. 40). 
